\title{
Prognostic Factors in Oral Cavity and Oropharyngeal Squamous Cell Carcinoma
}

\section{The Impact of Tumor-Associated Macrophages}

\author{
Benjamin Marcus, M.D. ${ }^{1}$ \\ Douglas Arenberg, M.D. ${ }^{2}$ \\ Julia Lee, M.s. ${ }^{3}$ \\ Celina Kleer, M.D. ${ }^{4}$ \\ Douglas B. Chepeha, M.D., M.S.P.H. ${ }^{1}$ \\ Cecelia E. Schmalbach, M.D., M.S. ${ }^{1}$ \\ Mozaffarul Islam, Ph.D. ${ }^{1}$ \\ Supriti Paul, M.D. ${ }^{1}$ \\ Quintin Pan, Ph.D. ${ }^{5}$ \\ Samir Hanash, M.D., Ph.D. ${ }^{6}$ \\ Rork Kuick, M.s. ${ }^{6}$ \\ Sofia D. Merajver, M.D., Ph.D. ${ }^{5}$ \\ Theodoros N. Teknos, M.D. ${ }^{1}$
}

\begin{abstract}
${ }^{1}$ Department of Otolaryngology-Head and Neck Surgery, University of Michigan Medical Center, Ann Arbor, Michigan.

${ }^{2}$ Department of Internal Medicine, Division of Pulmonary Medicine, University of Michigan Medical Center, Ann Arbor, Michigan.

${ }^{3}$ Department of Biostatistics, University of Michigan Medical Center, Ann Arbor, Michigan.
\end{abstract}

${ }^{4}$ Department of Pathology, University of Michigan Medical Center, Ann Arbor, Michigan.

${ }^{5}$ Department of Internal Medicine, Division of Hematology/Oncology, University of Michigan Medical Center, Ann Arbor, Michigan.

${ }^{6}$ Department of Pediatrics, University of Michigan Medical Center, Ann Arbor, Michigan.

Supported by a Specialized Programs of Research Excellence (SPORE) Grant in Head and Neck Cancer (National Institutes of Health, National Cancer Institute, National Institute of D Cancer Research NIDCR Grant 1P50 CA/DE97248-01) to Theodoros N. Teknos and Sofia D. Merajver.

Address for reprints: Theodoros N. Teknos, M.D., University of Michigan Medical Center, 1500 E. Medical Center Drive, 1904 Taubman Center, Ann Arbor, Ml 48103; Fax: (734) 936-9625; E-mail: llocke@umich.edu

Received May 7, 2004; revision received September 3, 2004; accepted September 3, 2004.

\begin{abstract}
BACKGROUND. The survival of patients with head and neck squamous cell carcinoma (HNSCC) remains unaffected despite recent therapeutic advances. To reverse this trend, reliable and clinically applicable markers of tumor aggressiveness must be identified. One such marker may be the tumor-associated macrophage content. The authors hypothesized that tumor-associated macrophages contribute to HNSCC aggressiveness, and the objective of the current study was to prove this hypothesis using mRNA expression analysis and a large cohort of clinical specimens.
\end{abstract}

METHODS. Oligonucleotide microarray analysis was performed on a prospective cohort of 20 patients with previously untreated oral cavity or oropharynx squamous cell carcinoma (OC/OP SCCA) and on normal oropharyngeal mucosa from 4 patients. After determining whether macrophage chemoattractants were produced by tumors, conditioned media from three HNSCC cell lines were used to quantify macrophage migration in an in vitro assay. A high-density tissue microarray of 102 patients with previously untreated OC/OP SCCA was stained immunohistochemically for CD68 to identify tissue macrophages, and the results were correlated with clinicopathologic data and survival.

RESULTS. Monocyte chemoattractant protein 1 was up-regulated significantly in tumors compared with normal mucosa $(P=0.0025$; fold change $=1.89$ ). All University of Michigan SCC tumor cell line conditioned media caused a significant increase in macrophage migration $(P<0.05)$. Tissue microarray data revealed that macrophage content of the primary tumor was associated strongly with lymph node metastasis $(P<0.0001)$, extracapsular lymph node spread $(P=0.0001)$, and advanced clinical disease stage $(P=0.0002)$. When it was evaluated along with other clinicopathologic data, the macrophage content was found to be an independent predictor of lymph node metastasis $(P<0.0001)$.

CONCLUSIONS. Primary tumor macrophage content is a strong predictor of tumor aggressiveness in HNSCC. Cancer 2004;101:2779-87.

(C) 2004 American Cancer Society.

KEYWORDS: high-density tissue microarray, migrated monocytes, monocyte chemoattractant protein 1, tumor aggressiveness, squamous cell carcinoma, head and neck cancer.

$H^{2}$ ead and neck squamous cell carcinoma (HNSCC) will affect 55,000 Americans and will result in 13,000 deaths this year alone. Advances in both surgical and nonsurgical therapeutics have led to increased local tumor control in recent years. However, overall mortality rates have not improved due to tumor recurrences at regional and distant sites of metastasis. To impact the survival of these patients, molecular and histologic markers must be identified that will help target tumors with a propensity for metastatic spread. Treat- 
ments tailored to these specific tumor determinants may reduce metastasis and thus enhanced cure rates. To date, no reliable or clinically applicable marker of tumor aggressiveness has been identified for HNSCC.

One such putative marker of tumor aggressiveness that has been identified in other malignancies is leukocytic infiltration of the tumor, commonly known as tumor-related leukocytosis (TRL). ${ }^{1}$ Over a 7 -year period Kasuga et al. investigated 227 patients with lung carcinoma. ${ }^{1}$ Among these patients, 33 tumors, which were predominately nonsmall cell lung carcinoma (NSCLC), were identified with TRL on pathologic examination. These patients also had elevated levels of serum granulocyte-macrophage-colony stimulating factor, interleukin 6 , and granulocyte-colony stimulating factor. It is interesting to note that patients with TRL had a significantly reduced overall survival compared with patients who had similarly staged tumors without TRL.

The pathophysiology and mechanism by which TRL and, more specifically, monocytosis effects tumor behavior may be related to a substantial increase in tumor angiogenesis. White al. ${ }^{2}$ showed in NSCLC that tumor cocultured with peripheral blood monocytes produced dramatically more proangiogenic cytokines compared with tumor that was grown alone. Endothelial cell migration also was enhanced by the conditioned media from the cocultured cells compared with tumor cells alone. Furthermore, it has been demonstrated that macrophages are important independent sources of proangiogenic cytokines on lung carcinoma. Synthesizing the available data, we hypothesized that tumor-associated macrophages (TAMs) contribute to HNSCC aggressiveness. To evaluate this hypothesis, we performed gene microarray expression analysis on fresh tumor specimens. After confirming the up-regulation of genes that are monocyte chemoattractants, functional studies were performed using conditioned media from well established HNSCC cell lines to investigate their effect on monocyte migration. Finally, the impact of TAMs on tumor aggressiveness and patient survival was evaluated using a large tissue microarray with extensive clinical/pathologic data and follow-up.

\section{METHODS AND MATERIALS Tissue Samples and Cell Lines}

A prospective cohort of 20 patients with newly diagnosed, previously untreated oral cavity (OC)/oropharyngeal (OP) squamous cell carcinoma (SCC) who presented to the University of Michigan Medical Center between February 2001 and December 2001 was investigated by mRNA expression analysis. All patients underwent primary tumor resection and cervical lymphadenectomy, and pathologic staging was performed. There were 7 AJCC Stage I-II tumors (lymph node negative tumors) and 13 Stage III-IV tumors (lymph node positive). At the time of surgical resection, a sample of the primary tumor was snap frozen in liquid nitrogen. Samples were embedded in optimal cutting temperature freezing media (OCT; Miles Scientific, Naperville, IL) and stored at $-80{ }^{\circ} \mathrm{C}$ for RNA extraction. A surgical pathologist evaluated a cryotome section $(5 \mu \mathrm{m})$ from each block using hematoxylin and eosin staining. Areas of tissue containing at least $70 \%$ tumor cellularity were selected for RNA isolation. Normal controls for the array studies included OC mucosa samples from 4 patients without cancer who underwent surgical treatment for obstructive sleep apnea and primary cell explants of OC keratinocytes.

\section{RNA Isolation}

Total cellular RNA was extracted from the tumor samples and cell lines as described previously. ${ }^{3}$ Samples were homogenized using Trizol reagent (Life Technologies, Gaithersburg, MD) and purified according to the manufacturer's instructions. RNA samples were purified further on RNeasy spin columns (Qiagen, Valencia, CA). RNA quality was assessed by $1 \%$ agarose gel electrophoresis in the presence of ethidium bromide. $^{3}$

\section{Microarray Analysis}

Preparation of cRNA and hybridization to commercially available HG_U95Av2 microarrays (Affymetrix, Santa Clara, CA) was performed according to the manufacturer's protocols, as outlined previously. ${ }^{3-14}$ These were general-purpose arrays that were not designed for any particular subset of genes. Microarrays were then scanned, and probe intensities were extracted from the image (GeneArray scanner and Microarray Suite 4.0; Affymetrix). Each probe set on the array typically consisted of 16 individual, 25-base oligonucleotide features complementary to specific cDNA, called perfect match (PM) probes, and 16 identical probes with a sequence that had been altered at the central base, called mismatch (MM) probes. Publicly available software was used to process the probe intensities to obtain normalized results. ${ }^{14}$ Probe pairs for which PM-MM $<-200$ on the standard were removed from the analysis, and the remaining PMMM differences were averaged for each probe set on each microarray by discarding the $25 \%$ highest and lowest differences and averaging the remaining differences. The intensities for each microarray were normalized to the standard using a piece-wise linear function that made 99 evenly spaced quintiles agree 
with the corresponding quantiles in the distribution of the standard. Fold changes were computed as the ratio of group means after first replacing means that were $<100 \times 100$. Normalized intensities were $\log$ transformed by mapping $\mathrm{x}$ to $\log (\max (\mathrm{x}+100,0)$ +100 ) prior to performing statistical tests. One-sided signed-rank tests of PM-MM differences for each probe set on each array were used to determine the number of detectable transcripts.

\section{Conditioned Media and Chemotaxis Assays}

University of Michigan (UM) head and neck carcinoma cell lines UM-SCC 9, 11b, and 38 were grown in standardized conditions $\left(1 \times 10^{6}\right.$ cell per flask, $37^{\circ} \mathrm{C}$, $5 \% \mathrm{CO}_{2}$, conditioned PBM media) until confluent. Media from the flask was flash frozen in $5 \mathrm{~mL}$ aliquots at $-20^{\circ} \mathrm{C}$. Media was thawed on ice immediately before single use.

\section{Chemotaxis assay}

In vitro monocyte chemotaxis was performed in modified Boyden chambers (Neuroprobe, Cabin John, MD), as described previously. ${ }^{4}$ Peripheral blood mononuclear cells were isolated from heparinized whole blood using Ficoll-Hypaque gradient centrifugation. The bottom wells of blind well chemotaxis chambers were filled with either 1) conditioned media from each cell line treated with control immunoglobulin G (IgG) antibody (1:500 dilution) or 2) conditioned media treated with anti-CCR2 antibody. Filters were placed over the wells, and the top wells were filled with freshly isolated human peripheral blood mononuclear cells (normal nonsmoking donor; 3 $\times 10^{6}$ cells $\left./ \mathrm{mL}\right) .{ }^{4}$ Chambers were placed in humidified, $37{ }^{\circ} \mathrm{C}, 5 \% \mathrm{CO}_{2}$ incubators for 1.5 hours. Filters were then removed, stained, and mounted on slides. Migrated cells were counted in five high-power fields (HPF) per well. Each condition was run in triplicate, and the experiments were repeated three times. Results were expressed as the mean \pm standard error of the mean of cells per HPF.

\section{Immunohistochemical staining of the tissue microarray}

The validation of increased macrophage content in metastatic tumors was investigated using immunohistochemical analysis on a tissue microarray comprised of 102 independent patient tumor samples. All patients for this validation group presented to the University of Michigan Hospital between 1997 and 2000 with newly diagnosed, previously untreated OC and OP SCC (Table 1). None of the patients from the gene array portion of the study were included in the tissue microarray validation group. All patients underwent pathologic staging after surgical tumor resection and
TABLE 1

Descriptive Statistics on Demographic and Clinical Variables

\begin{tabular}{|c|c|c|}
\hline Variable & Value & $95 \% \mathrm{CI}$ \\
\hline Total no. of patients & 102 & - \\
\hline \multicolumn{3}{|l|}{ Age (yrs) } \\
\hline Mean \pm SD & $56.3 \pm 14.6$ & - \\
\hline Median & 56.0 & $53.4-59.2$ \\
\hline Range & $33-74$ & - \\
\hline Median follow-up (mos.) & 41.1 & $36.0-45.3$ \\
\hline No. of male patients (\%) & $76 / 102(74.5)$ & $66.1-83.0$ \\
\hline No. with positive lymph nodes (\%) & $69 / 102(67.7)$ & $58.6-76.7$ \\
\hline \multicolumn{3}{|l|}{ No. with each no. of positive lymph nodes (\%) } \\
\hline 0 & $33 / 102(32.4)$ & - \\
\hline 1 & $21 / 102(20.6)$ & - \\
\hline 2 & $13 / 102(12.80)$ & - \\
\hline 3 & $10 / 102(9.8)$ & - \\
\hline$>3$ & $25 / 102(24.5)$ & - \\
\hline No. with ECS (\%) & $40 / 102(39.2)$ & $29.7-48.7$ \\
\hline No. with early T classification (T1-T2) (\%) & $37 / 100(37.0)$ & $27.5-46.5$ \\
\hline \multicolumn{3}{|l|}{ No. each with T classification (\%) } \\
\hline $\mathrm{T} 0$ & $2 / 102(2.0)$ & - \\
\hline $\mathrm{T} 1$ & $8 / 102(7.8)$ & - \\
\hline $\mathrm{T} 2$ & $29 / 102(28.4)$ & - \\
\hline $\mathrm{T} 3$ & $27 / 102(26.5)$ & - \\
\hline $\mathrm{T} 4$ & $36 / 102(35.3)$ & - \\
\hline No. with perineural invasion (\%) & 19/98 (19.4) & $11.6-27.2$ \\
\hline No. with clinical Stage IV disease (\%) & $62 / 102(60.8)$ & $51.3-70.3$ \\
\hline \multicolumn{3}{|l|}{ No. in each clinical stage (\%) } \\
\hline Stage 1 & 6/102 (5.9) & - \\
\hline Stage 2 & $16 / 102(15.7)$ & - \\
\hline Stage 3 & $18 / 102(17.7)$ & - \\
\hline Stage 4 & $62 / 102(60.8)$ & - \\
\hline \multicolumn{3}{|c|}{ No. with each type of primary differentiation (\%) } \\
\hline Well & $14 / 96(14.6)$ & - \\
\hline Moderate & $60 / 96(62.5)$ & - \\
\hline Poor & $22 / 96(22.9)$ & - \\
\hline Three-yr overall survival rate (\%) & 64.6 & $54.9-74.4$ \\
\hline
\end{tabular}

SD: standard deviation; 95\% CI: 95\% confidence interval; ECS: extracapsular spread.

lymphadenectomy (69 lymph node-positive tumors and 33 lymph node-negative tumors). The mean age was 57 years in the lymph node-positive validation group and 58 years in the lymph node-negative group. The mean follow-up was 22 months in the lymph node-positive group and 28 months in lymph nodenegative group. The mean disease-free interval was 20 months for the lymph node-positive group and 28 months for lymph node-negative group. Significant tobacco and alcohol exposure was present in both groups. A head and neck pathologist identified and marked representative areas of tumor and normal squamous mucosa from each patient's paraffin-embedded primary tumor block. A high-density tissue microarray was constructed from each individual block using 3 replicate tumor cores $(0.6 \mathrm{~mm}$ in greatest dimension) and 1 normal mucosa core per patient. Cryotome sections $(5 \mu \mathrm{m})$ were then cut from the 
high-density tissue microarray and stained for CD68. The slides were placed in TBST, and immunohistochemistry was performed using the Envision + Mouse kit from Dako Cytomation (Dako Corporation, Carpinteria, CA). The CD68 primary antibody (Dako Cytomation) was incubated for 30 minutes at room temperature at a dilution of 1:1600. Appropriate positive and negative isotype control antibodies were utilized. Utilizing digital image capture, each core of the tissue microarray was examined after staining for CD68. All core samples were analyzed by a single examiner who was blinded with regards to each patient's clinical information. Cells were considered positive if cytoplasmic staining was observed. Representative cores were reviewed with a head and neck pathologist for confirmation of analysis. Each core was then given an absolute count for the number of cells that were positive for CD68 (representing macrophages).

\section{Statistical Analysis}

Because of multiple core measurements per patient, the macrophage content is expressed as the weighted mean \pm standard deviation, in which the weight is the reciprocal of patient-wise, nonmissing macrophage data points. The covariates of interest included the metastasis of tumor to lymph nodes (positive vs. negative), the presence of lymph node tumor with extracapsular spread (ECS), T classification (T0, T1, T2, T3, or T4), clinical stage (Stage I, II, III, or IV), perineural invasion (presence vs. absence), primary differentiation (poorly, moderately, or well differentiated), tobacco use (yes vs. no), alcohol use (yes vs. no), comorbidities (as measured by the Charleston comorbidity index), age, gender, overall survival, and disease-free survival.

To evaluate the association of macrophages with covariates of interest, generalized linear models were fit to the data using a generalized estimating equation approach to account for both within-patient and between-patient variation derived from repeated macrophage measurements. A Poisson distribution was assumed for macrophage counts. Over-dispersion was corrected by a scale parameter that was estimated by dividing the square root of the Pearson chi-square statistic by degrees of freedom. Each covariate of interest (except survival outcomes) was tested univariately in the model as a main effect. The same analysis was conducted on a subgroup of patients with early $\mathrm{T}$ classification ( $\mathrm{T} 1$ and $\mathrm{T} 2$ ).

Logistic regression was used to study the relation between lymph node metastases (positive vs. negative) and macrophage content along with all the demographic and clinical variables of interest, including age, gender, comorbidities, alcohol use, tobacco use, perineural invasion, primary differentiation, T classification, and clinical stage. Due to multicollinearity, only $\mathrm{T}$ classification or clinical stage was included in the model. The patient-wise median macrophage count was used in the logistic regression model.

The predictive value of macrophage content on survival outcomes was explored by the Kaplan-Meier method and the log-rank test. The patient-wise median macrophage count was used in this analysis. In addition, the log-rank test was used to compare the homogeneity of survival rate between each category of discrete clinical variables.

All statistical analyses were performed using SAS software (version 8.2; SAS Institute Inc., Cary, NC). A two-tailed $P$ value $\leq 0.05$ was considered statistically significant. No multiple comparison adjustments were made.

\section{RESULTS \\ Microarray Analysis}

Using the Affymetrix HG_U95Av2 GeneChip (which consisted of 12625 probe sets representing approximately 9600 genes), we obtained gene expression profiles for 13 OC/OP SCC tumors that were metastatic to cervical lymph nodes (lymph node positive), 7 nonmetastatic OC/OP SCC tumors (lymph node negative), and 4 normal OC mucosa samples and normal OC keratinocyte explants. Tumor samples averaged 7731 detectable probe sets, and normal samples averaged 7798 detectable probe sets $(P<0.05)$. We identified 223 probe sets that represented angiogenesis-related genes by reference to the literature and by searching for the Gene Ontology term angiogenesis applied to genes on LocusLink (available from URL: http:// www.ncbi.nlm.nih.gov/LocusLink/[accessed 8/30/2004]). Two-sample Student $t$ tests using log-transformed data from tumor specimens versus normal mucosa were performed specifically comparing expression profiles of these 223 probe sets. Sixty-six probe sets met statistical significance at a $P$ value $<0.05$ and with a fold change of 1.5. We randomly permuted the sample labels 1000 times using the same criteria on these 1000 data sets and obtained an average of only 7.1 qualifying probe sets. Fifty-two probe sets (40 genes) were up-regulated in tumors compared with normal mucosa controls (Fig. 1). Included in these were 3 probes sets that represented small inducible cytokine A2, also know as monocyte chemotactic protein 1 (MCP-1).

\section{Chemotaxis Assays}

From our microarray data, the gene for MCP-1 was expressed to a much greater degree in patients with head and neck carcinoma than in normal tissue. To 
FIGURE 1. This heat map illustrates relative gene expression of the 66 probe sets that were expressed differentially between the patient's primary tumors and normal mucosa (represents 51 genes, including 40 up-regulated genes and 11 down-regulated genes). Probe sets yielded $P$ values $<0.05$ (Student $t$ test), and fold-changes $>1.5$ are shown for tumor versus normal mucosa.
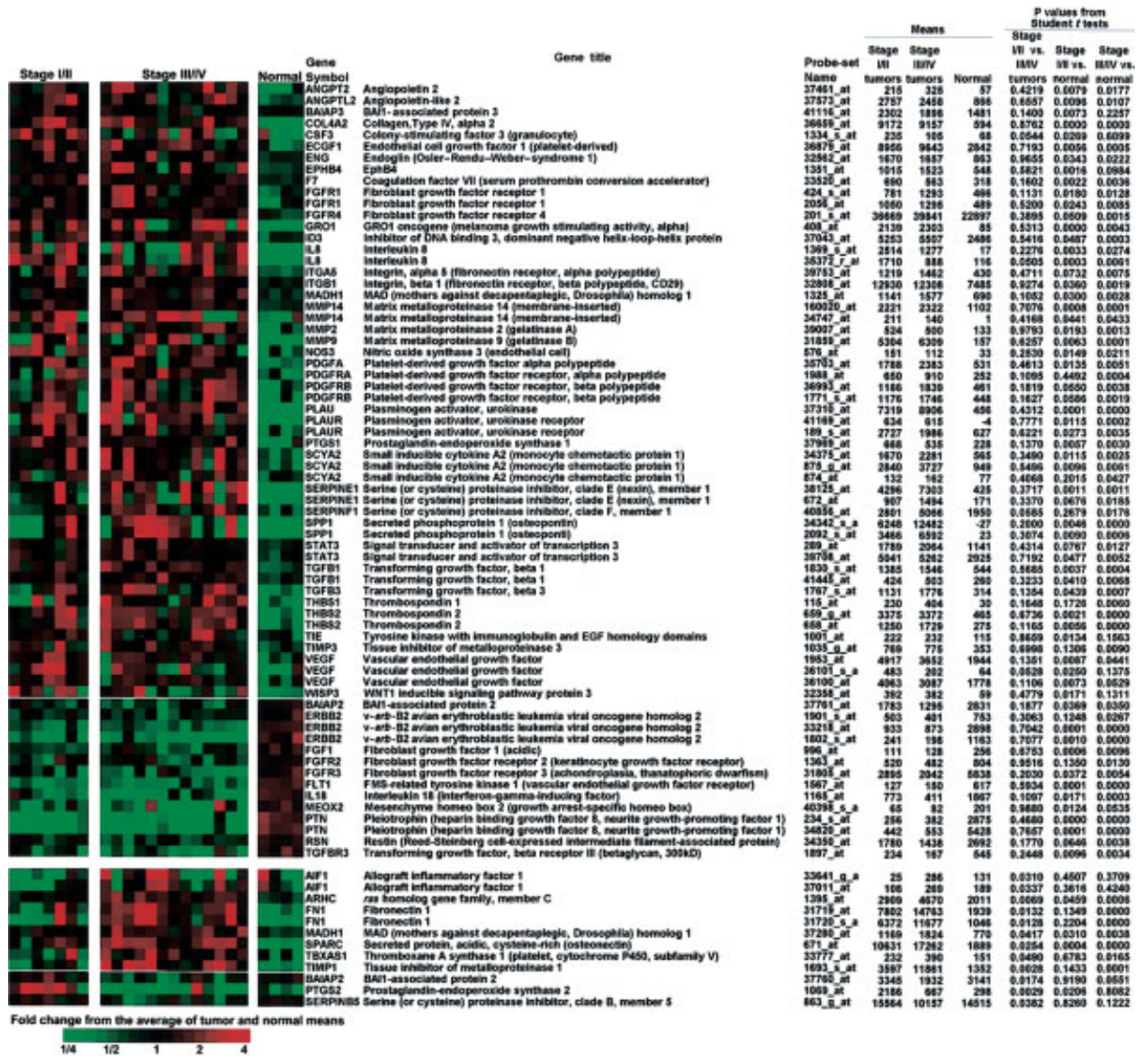

test the functional significance of this observation, we evaluated whether head and neck carcinoma attracts monocytes in vitro. We used UM-SCC cell lines 9, 11b, and 38 and tested their attraction potential in standard monocyte chemotaxis assays. Standardized controls determined a baseline chemotaxis of $3.2 \pm 0.61$ cells per HPF. Tumor conditioned media-induced chemotaxis was observed at an average of $17.3 \pm 3.05$ cells per HPF. In the presence of the control antibody (IgG) UM-SCC 9 induced $13.0 \pm 1.37$ cells per HPF, UM-SCC $11 \mathrm{~b}$ produced a migration of $29.1 \pm 4.15$ cells per HPF, and UM-SCC 38 demonstrated a chemotaxis of $39.9 \pm 8.7$ cells per HPF. When the conditioned media was treated with anti-CCR2 antibody (blocks the MCP-1 receptor), UM-SCC $11 \mathrm{~b}$ and 38 had a significant decrease in monocyte chemotaxis compared with the control antibody (Fig. 2).

\section{Tissue Microarray and CD68 Staining}

After illustrating increased genetic expression of monocyte chemoattractant factors as well as increased monocyte chemotaxis induced by conditioned media from HNSCC cell lines, we stained the high-density tissue microarray to investigate whether these factors were correlated with primary tumor macrophage content and with relevant clinical parameters (Fig. 3). The clinical parameters available for analysis included 1) the presence of metastatic lymph nodes, 2) the number of metastatic lymph nodes, 3) the presence of extracapsular spread, 4) the T classification of the tumor, 5) the overall tumor stage, 6) the presence of perineural spread, 7) the patient's age, 8) comorbidities, 9) tobacco use, 10) alcohol use, 11) gender, and 12) tumor differentiation. Sixty-nine of the 102 patients had positive lymph node status, and 33 patients had negative lymph node status. There were 164 and 84 primary tumor cores available from patients with positive and negative lymph node status, respectively. Among 102 patients, 55 patients had normal core data available. Among the most significant findings were the following: 1) There was a statistically significant difference $(P<0.0001)$ in the macrophage contents between normal tissue and tumor tissue. The average macrophage content was $11.1 \pm 14.6$ and 38.0 \pm 27.8 macrophages per HPF for normal cores and tumor cores, respectively. 2) There was a marked difference $(P<0.0001)$ in the macrophage content of primary tumor tissue between patients with positive 


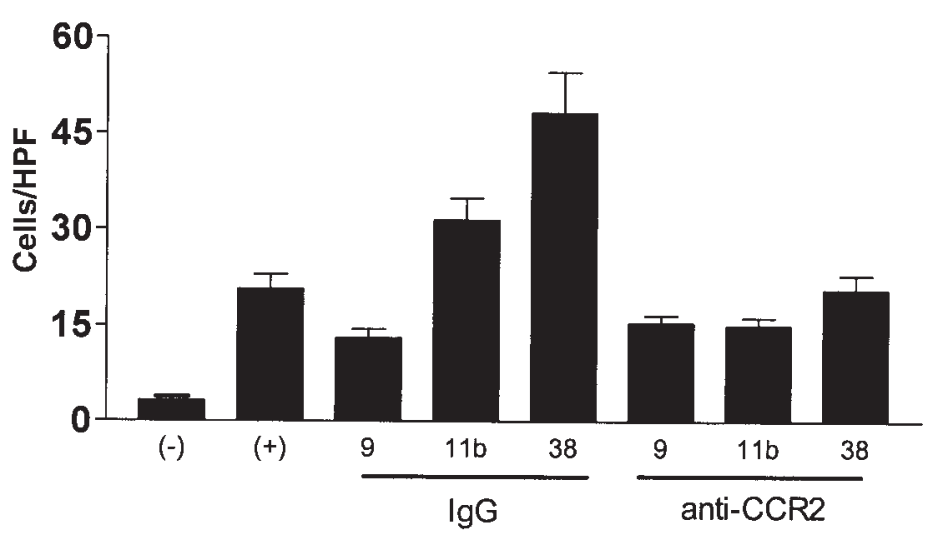

FIGURE 2. Chemotaxis assay: The $x$-axis represents the type of University of Michigan head and neck squamous cell carcinoma cell line assayed with control antibody (immunoglobulin G [lgG]) or with chemokine-inhibiting antibody (anti-CCR2), which is a known inhibitor of monocyte chemoattractant protein 1 . The $y$-axis represents the number of migrated monocytes per high-power field.

A
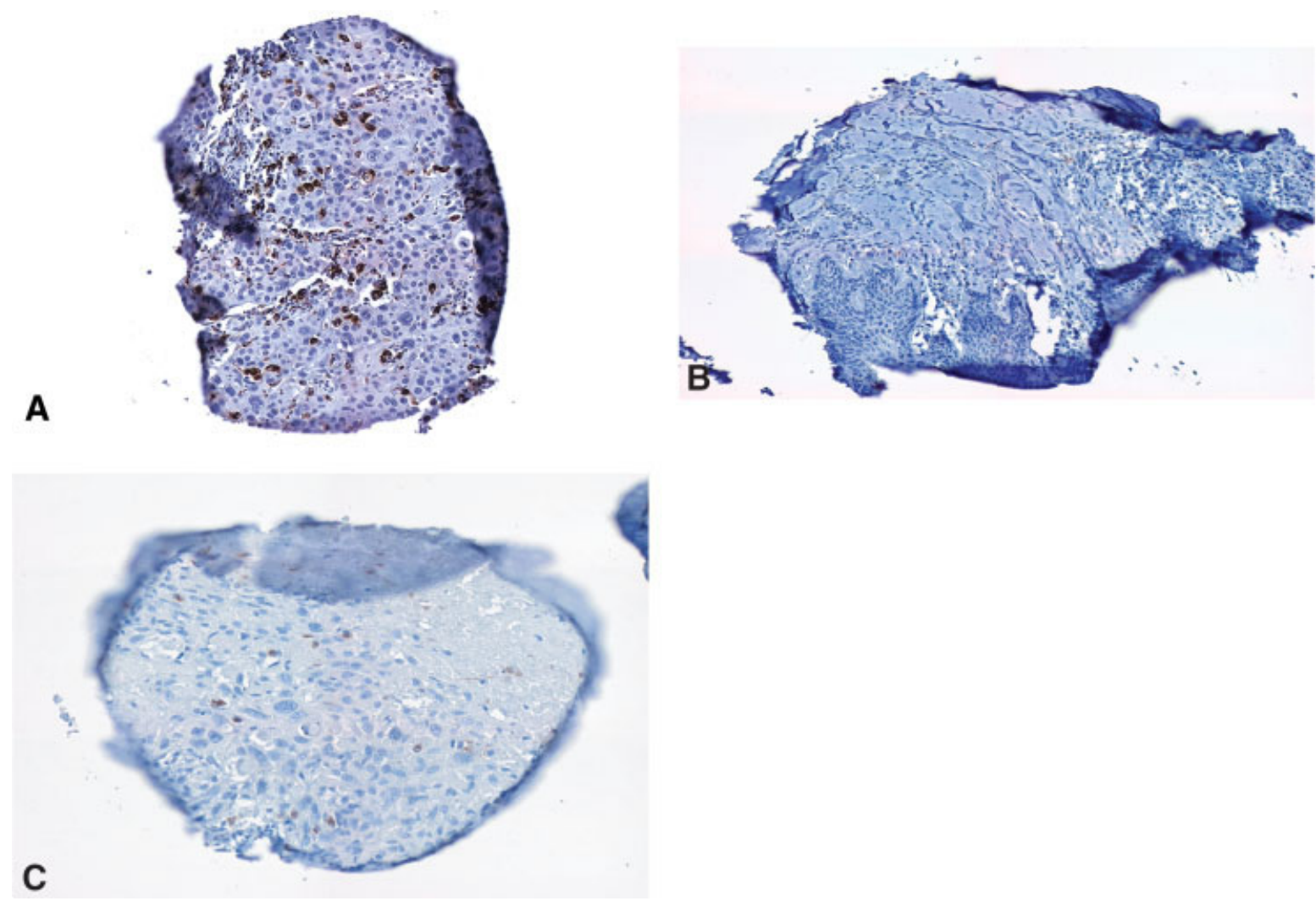

FIGURE 3. (A) This sample of tumor was stained with CD68 for macrophages from a patient with known positive lymph node status. (B) This sample of normal mucosa tissue was stained with CD68 for macrophages. (C) This sample of tumor from a patient with negative lymph node status was stained with CD68 for macrophages.

lymph nodes and patients with negative lymph nodes. The average macrophage content of tumor cores from patients with positive lymph nodes and patients with negative lymph nodes were $46.7 \pm 27.9$ macrophages per HPF and $21.0 \pm 15.2$ macrophages per HPF, respectively. 3) Elevated macrophage counts in the primary tumor were correlated with lymph node metastases $(P<0.0001)$, lymph node ECS $(P=0.0001)$, advanced clinical stage $(P=0.0002)$, and alcohol use $(P=0.003) .4)$ When the macrophage count was eval- uated along with the other clinical parameters mentioned above, macrophage content was found to be an independent predictor of lymph node metastasis $(P$ $<0.0001)$. The subgroup analysis of patients with $\mathrm{T} 1$ and T2 tumors ( $n=36$ patients) demonstrated a similar pattern of statistical significance. Specifically, the primary tumor core macrophage content was higher in patients who had positive lymph nodes compared with patients who had negative lymph nodes $(P$ $=0.001)$, in patients who had ECS compared with 
TABLE 2

Correlations with Elevated Macrophage Counts at the Primary Tumor Site

\begin{tabular}{ll}
\hline Elevated macrophage count correlated with & $P$ value \\
\hline Presence of metastatic lymph nodes & $<0.0001$ \\
Presence of extracapsular spread & 0.0001 \\
Advanced clinical stage & 0.002 \\
Alcohol use & 0.003 \\
Lymph node metastasis in small primary tumors (T1, T2) & 0.001 \\
Extracapsular spread in small primary tumors (T1, T2) & 0.03 \\
\hline
\end{tabular}

patients who had no ECS $(P=0.03)$, in patients who had a later clinical disease stage compared with patients who had an earlier clinical disease stage $(P$ $=0.03$ ), and in patients who used alcohol compared with patients who did not use alcohol $(P=0.03)$. Clearly, all these findings suggest that primary tumor macrophage content correlates with aggressive tumor behavior, even in small tumors, manifesting with increase lymph node metastasis and an increased incidence of ECS (Table 2).

\section{Survival Analysis}

Despite correlations with many poor prognostic factors, the macrophage count did not appear to correlate directly with disease-specific survival, overall survival, or time to disease recurrence. Disease-free survival was affected negatively by lymph node metastases $(P=0.03)$, lymph node ECS $(P=0.02)$, advanced clinical stage $(P=0.04)$, advanced T classification $(P=0.02)$, and primary tumor perineural invasion $(P=0.02)$. Similarly, overall survival was affected negatively by lymph node metastases $(P$ $=0.04)$, lymph node ECS $(P=0.04)$, primary tumor perineural invasion $(P=0.03)$, advanced T classification $(P=0.005)$, and advanced clinical stage $(P$ $=0.01)$. Finally, variables that were found to be correlated with a shorter time to disease recurrence included lymph node metastases $(P=0.03)$, ECS $(P$ $=0.02)$, primary tumor perineural invasion $(P=0.02)$, and clinical stage $(P=0.04)$. Factors that were correlated with overall survival, disease-specific survival, and time to disease recurrence are summarized in Table 3.

\section{DISCUSSION}

In the current study, we identified clinical and histologic features that are predictive of decreased survival in patients with OC/OP SCC. We also demonstrated a strong correlation between primary tumor macrophage content and several of these predictive clinical markers. Furthermore, our gene microarray analysis
TABLE 3

The Effect of Various Clinical and Histologic Parameters on Survival and Recurrence in Patients with Oral Cavity and Oropharyngeal Squamous Cell Carcinoma

\begin{tabular}{llll}
\hline & \multicolumn{2}{c}{$\boldsymbol{P}$ value } \\
\cline { 2 - 4 } & & & Time to \\
Clinical parameter & OS & DFS & recurrence \\
\hline Lymph node metastasis & $0.04^{\mathrm{a}}$ & $0.03^{\mathrm{a}}$ & $<0.0001^{\mathrm{a}}$ \\
Age & 0.24 & 0.67 & 0.56 \\
Total comorbidities & 0.31 & 0.77 & 0.87 \\
Tobacco use & 0.07 & 0.14 & 0.10 \\
Alcohol use & 0.38 & 0.48 & 0.42 \\
Gender & 0.37 & 0.33 & 0.25 \\
Extracapsular spread & $0.04^{\mathrm{a}}$ & $0.02^{\mathrm{a}}$ & $0.02^{\mathrm{a}}$ \\
Tumor differentiation & 0.48 & 0.10 & 0.16 \\
Tumor perineural invasion & $0.03^{\mathrm{a}}$ & $0.02^{\mathrm{a}}$ & $0.02^{\mathrm{a}}$ \\
Tumor lymphatic invasion & 0.12 & $0.04^{\mathrm{a}}$ & 0.07 \\
T classification & $0.005^{\mathrm{a}}$ & $0.02^{\mathrm{a}}$ & 0.17 \\
Clinical stage & $0.01^{\mathrm{a}}$ & $0.01^{\mathrm{a}}$ & $0.04^{\mathrm{a}}$ \\
\hline
\end{tabular}

OS: overall survival; DFS: disease-free survival; TTR: time to recurrence.

${ }^{\text {a }}$ Statistically significant finding.

and monocyte chemotaxis assays illustrate a propensity for head and neck SCC to attract macrophages to the primary tumor bed. Based on the presented data, it is clear that macrophages increase head and neck tumor aggressiveness. The mechanism for this effect is elusive but is based on emerging evidence: It appears that an increase in tumor angiogenesis may be a critical component.

It is well known that tumors that are unable to create neovascularization are unable to grow beyond the critical size of $2-3 \mathrm{~mm} .{ }^{5}$ This angiogenesis and resultant tumor growth is essential for subsequent metastasis. ${ }^{5}$ A number of factors have been identified as central to angiogenesis, including both proangiogenic and antiangiogenic factors. Previous work has shown that macrophages, in addition to the tumor itself, serve as a source of proangiogenic cytokines. ${ }^{6}$ Thus, the infiltration of TAMs may be an important step necessary for tumors to achieve critical size and metastasis. Although it has been shown that macrophages in isolation are capable of proangiogenic activity, Polverini ${ }^{7}$ demonstrated that macrophages associated with tumor microenvironments are significantly more active in their cytokine production. Therefore, the synergy between tumor and monocytes creates a dramatic proangiogenic tumor phenotype. Further solidifying this concept, Arenberg et al. examined the behavior of TAM in NSLC. ${ }^{4}$ Histologic analysis of representative NSCLC revealed significant presence of infiltrating macrophages. After confirming tumor-driven monocyte infiltration, Arenberg et al. 
also demonstrated that homogenates of their NSCLC tumors induced significant monocyte chemotaxis and production of proangiogenic CXC chemokines. Possibly the most convincing work illustrating the importance of TAM in tumor progression and metastases published to date comes from the work of Webb et al., Scholl et al., Sieuwerts et al., and Lin et al. ${ }^{8-11}$ Using transgenic mouse technology, these investigators were able to create colony-stimulating factor 1 (CSF-1) null mutant mice, which had a relative absence of mature macrophages in many tissues, including the mammary gland. In their studies, these researchers illustrated that the absence of macrophages did not affect tumor formation and growth at the primary tumor site (breast), but metastases of these tumors to the lungs was significantly delayed. Furthermore, the restoration of macrophage infiltration with expression of CSF-1 restored macrophage infiltration at the primary tumor site and dramatically accelerated tumor progression and metastases. ${ }^{11}$ Subsequent clinical studies in breast carcinoma also have shown that marked macrophage infiltration in these tumors is correlated with a poor prognosis due to increased cytokine-mediated angiogenesis. ${ }^{12}$

Another potential mechanism by which TAM may lead to increased tumor aggressiveness is by means of altered antigen presentation. In recent years, it has become increasingly evident that low numbers of mature dendritic cells in head and neck tumors are correlated with poor clinical outcomes. ${ }^{15}$ Furthermore, in both renal cell carcinoma and melanoma, primary tumors with a high macrophage content behaved more aggressively and had lower numbers of activated dendritic cells within the tumor itself. ${ }^{16,17}$ These results suggest that the tumor microenvironment is altered by macrophages in such a way that it fails to initiate the expected protective activation of dendritic cells. Although to our knowledge this issue has not been studied to date in SCC of the head and neck, this is a very intriguing question and one that we will be addressing in the near future.

In the current study, we demonstrated a correlation between aggressive tumor behavior in head and neck carcinoma and the level of primary tumor macrophage infiltration. Specifically, patients who had tumors with higher levels of macrophage infiltration were more likely to develop lymph node metastases as well as extracapsular spread of tumor through the lymph nodes. In fact, macrophage content was found to be an independent predictor of lymph node metastasis in a multivariable analysis of tumor and patient characteristics. Even more significant, however, was the finding that there was a correlation between TAMs and lymph node metastasis and ECS in T1 and T2 primary OC/OP tumors. However, it is interesting to note that even though there was strong correlation between the level of macrophage infiltration and the presence of lymph node metastases and ECS (two distinct and statistically significant negative prognostic factors), TAM levels were not correlated with overall survival, disease-specific survival, or time to disease recurrence. This may have been due to the relatively short follow-up in some of our patient cohort and, as the data base matures, a stronger correlation may emerge. A more likely explanation, however, is the fact that there was wide variety in the TAM count from patient to patient, and it is unlikely that a reliable "cut-off point" can be determined above which tumors are more likely to metastasize and below which they will not. Regardless, the data from the current study and others clearly indicate that macrophage infiltration plays a role in the aggressive tumor phenotype, and further investigations are warranted to identify the mechanisms of this effect. With further study, potential clinical application of these findings may involve assessing primary tumor macrophage content of early lesions as a method of determining the need for selective neck dissection or as a means of identifying patients with particularly aggressive tumors as candidates for neoadjuvant therapy.

\section{REFERENCES}

1. Kasuga I, Makino S, Ohyashinki K. Tumor related leukocytosis is linked with poor prognosis in patients with lung carcinoma. Cancer. 2001;92:2399-2405.

2. White E, Strom S, Wys NL, Arenberg D. Non-small cell lung cancer cells induce monocytes to increase expression of angiogenic activity. J Immunol. 2001;166:7549-7555.

3. Rickman DS, Bobek MP, Misek DE, et al. Distinctive molecular profiles of high-grade and low-grade gliomas based on oligonucleotide microarray analysis. Cancer Res. 2001;61: 6885-6891.

4. Arenberg DA, Keane MP, DiGiovine B, et al. Macrophage infiltration in human non-small cell lung cancer: the role of CC chemokines. Cancer Immunol Immunother. 2000;49:63-70.

5. Zetter BR. Angiogenesis and tumor metastasis. Annu Rev Med. 1998;49:407-424.

6. Kataki A, Scheid P, Piet M, et al. Tumor infiltrating lymphocytes and macrophages have a potential dual role in lung cancer by supporting both host-defense and tumor progression. J Lab Clin Med. 2002;140:320-328.

7. Polverini PJ. Role of the macrophage in angiogenesis-dependent diseases. EXS. 1997;79:11-28.

8. Webb SE, Pollard JW, Jones GE. Direct observation and quantification of macrophage chemoattraction to the growth factor CSF-1. J Cell Sci. 1996;109(Pt 4):793-803.

9. Scholl SM, Libereau R, de la Rochefordiére A, et al. Circulating levels of the macrophage colony stimulating factor CSF-1 in primary and metastatic breast cancer patients. A pilot study. Breast Cancer Res Treat. 1996;39:275-283. 
10. Sieuwerts AM, Klijn JG, Henzen-Logmans SC, Foekens JA. Cytokine-regulated urokinase-type-plasminogen-activator (uPA) production by human breast fibroblasts in vitro. Breast Cancer Res Treat. 1999;55:9-20.

11. Lin EY, Nguyen AV, Russell RG, Pollard JW. Colony-stimulating factor 1 promotes progression of mammary tumors to malignancy. J Exp Med. 2001;193:727-740.

12. Lewis CE, Leek R, Harris A, McGee JO. Cytokine regulation of angiogenesis in breast cancer: the role of tumor-associated macrophages. J Leukoc Biol. 1995;57:747-751.

13. Eisen MB, Spellman PT, Brown PO, Botsen D. Cluster analysis and display of genome-wide expression patterns. Proc Natl Acad Sci USA. 1998;95:14863-14868.

14. Schwartz DR, Kardia SLR, Shedden KA, et al. Gene expression in ovarian cancer reflects both morphology and biological behavior, distinguishing clear cell from other poor-prognosis ovarian carcinomas. Cancer Res. 2002;62:4722-4729.

15. Gallo O, Bianchi S, Giannini A, Gallina E, Libonati GA, FiniStorchi O. Correlations between histopathological and biological findings in nasopharyngeal carcinoma and its prognostic significance. Laryngoscope. 1991;101:487-493.

16. Troy AJ, Summers KL, Davidson PJ, Atkinson CH, Hart DN. Minimal recruitment and activation of dendritic cells within renal cell carcinoma. Clin Cancer Res. 1998;4:585-593.

17. Byrne SN, Halliday GM. Phagocytosis by dendritic cells rather than MHC II high macrophages is associated with skin tumour regression. Int $J$ Cancer. 2003;106:736744 . 